\title{
EDITORIAL
}

For reprint orders, please contact: reprints@futuremedicine.com

\section{Can the addition of verapamil to bedaquiline-containing regimens improve tuberculosis treatment outcomes? A novel approach to optimizing TB treatment}

“...with very few anti-tuberculosis drugs in the pipeline, repurposing existing approved drugs as adjuvants to shorten treatment duration has emerged as a promising alternative strategy to outpace the evolution of drug resistance.”
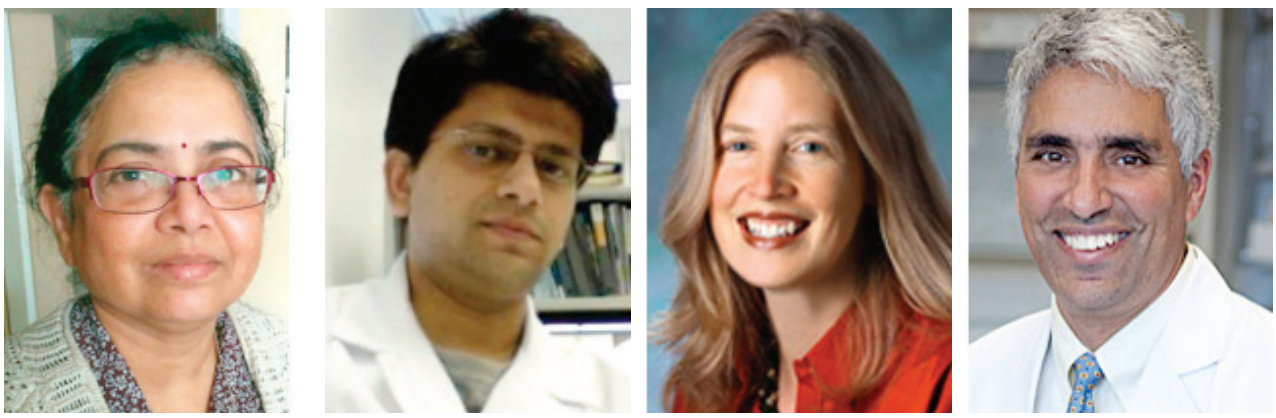

Geetha Srikrishna ${ }^{\dagger, 1,2,3}$, Shashank Gupta ${ }^{\dagger, 1,2,3}$, Kelly E Dooley ${ }^{1,2,4}$ \& William R Bishai ${ }^{* 1,1,2,3}$

Tuberculosis (TB) is the second leading cause of mortality due to a single infectious agent worldwide. Despite the availability of effective chemotherapy for more than 50 years, a combination of many factors, including poor adherence to the drug regimen, limited health system resources and immune system compromise from HIV co-infection, have contributed to the emergence and spread of highly transmissible, drug-resistant strains of Mycobacterium tuberculosis (M. tb). According to WHO, there were 9 million cases of TB in 2013, and approximately 480,000 cases of multidrug resistant TB (MDR-TB) (M. $t b$ with resistance to isoniazid and rifampin), the latter associated with an estimated 210,000 deaths. Extensively drug-resistant (XDR) and totally drug resistant (TDR) TB have become a global health threat. Moreover, many existing second-line drugs are highly toxic and poorly efficacious. The need for new drugs and tools to deal with the alarming spread of drug-resistant $\mathrm{TB}$, and to shorten the duration of treatment for drug-sensitive TB, is highly imperative. Against this backdrop, and with very few anti-TB drugs in the pipeline, repurposing existing approved drugs as adjuvants to shorten treatment duration

\section{KEYWORDS}

- adjunctive therapy $\bullet$ bedaquiline

- cardiotoxicity • drug-resistant tuberculosis • verapamil

'Center for Tuberculosis Research, Johns Hopkins University School of Medicine, Room 108, 1550 Orleans St, Baltimore, 


\section{"Co-administration of verapamil with \\ bedaquiline is a promising strategy for improving MDR-TB treatment efficacy and for reducing dose size requirements of bedaquiline; plus verapamil may be cardioprotective, reducing the risk of QT prolongation with bedaquiline and companion drugs.”}

has emerged as a promising alternative strategy to outpace the evolution of drug resistance.

\section{Efflux pump inhibitors \& tuberculosis}

Drug efflux pumps play a major role in drug resistance while serving other important functions in bacteria. Drug resistance may also emerge as a consequence of drug tolerance, which commonly occurs among bacterial 'persisters' that enter a nonreplicating state within the host and have reduced susceptibility to antibacterial drugs [1]. Adams et al. discovered one mechanism for drug tolerance with their work showing that mycobacteria $M$. marinum and $M$. th developed drug tolerance following infection of macrophages, a phenomenon that was attributed to bacterial efflux pumps whose expressions were induced upon macrophage infection [2]. Efflux pump inhibitors such as verapamil, reserpine and thioridazine may, therefore, help overcome this tolerance and enhance the activity of anti-TB drugs. Verapamil is an US FDA approved drug used to treat heart disease and hypertension. As proofof-concept, Adams et al. showed that verapamil indeed reduced macrophage-induced bacterial drug tolerance $[2,3]$. In a mouse model of TB, initial findings by Louw and colleagues showed that verapamil in combination with first-line drugs significantly reduced pulmonary bacterial counts after 2 months of treatment [4]. Verapamil also restored rifampin efficacy in mice infected with rifampin-resistant $M$. $t b$. We provided further compelling evidence that adding verapamil to standard chemotherapy not only reduced the time required to render TB-infected mouse lungs culture negative from standard 6 months to 4 months, but also significantly decreased the risk of relapse when assessed 3 months following treatment completion [5]. Our findings highlighted the potential of verapamil to augment the sterilizing activity of standard TB treatment. Our pharmacokinetic studies showed that the administration of a $12.5 \mathrm{mg} / \mathrm{kg}$ dose of verapamil to mice results in a maximum serum concentration $\left(\mathrm{C}_{\max }\right)$ of approximately $500 \mathrm{ng} / \mathrm{ml}$ and an AUC of $792 \mathrm{ng} \cdot \mathrm{h} / \mathrm{ml}$, close to the reported human AUC of $841 \mathrm{ng} \cdot \mathrm{h} / \mathrm{ml}$ when $240 \mathrm{mg}$ of verapamil (sustained release formulation) is administered with food [5]. A clinical trial with verapamil is currently underway in India to determine the safety and tolerability of verapamil in patients with $\mathrm{TB}$ without underlying cardiac disease, and to evaluate the pharmacokinetics of verapamil when administered in conjunction with rifampin [21].

\section{Introduction of bedaquiline}

In 2012, bedaquiline (Sirturo ${ }^{\circledR}$, also known as TMC207 or R207910) became the first anti-TB drug of a new class in more than 40 years to be approved by the FDA. It is approved specifically for patients with MDR-TB, under the provisions of the accelerated approval program for serious diseases. Bedaquiline is a diarylquinoline with a novel mechanism of action. It inhibits mycobacterial ATP synthase in actively replicating and nonreplicating drug-sensitive and drug-resistant strains of $M . t b$, thus disrupting the energy metabolism of mycobacteria [6]. Owing to its unique mechanism of action, there is minimal cross-interference with existing anti-TB drugs. In addition, human mitochondrial ATP synthase is 20,000 times less sensitive for bedaquiline than the mycobacterial enzyme, making it an attractive, novel anti-TB drug. In preclinical studies, bedaquiline was found to exhibit bactericidal activity [7]. When added to a second line background regimen in patients with newly diagnosed MDR-TB, bedaquiline improved sputum culture conversion after 8 weeks of treatment compared with placebo $[8]$. When administered for 24 weeks in a larger number of patients, bedaquiline reduced the median time to sputum culture conversion, from 125 days to 83 days when compared with placebo, and also increased the rate of culture conversion [9].

While efficacy data using bedaquiline have been very encouraging, safety concerns associated with the use of bedaquiline, including prolonged QTc interval on electrocardiogram, and unexplained increase in late mortality [9], have prompted the implementation of a black box warning on the drug label. The QTc interval represents the time required for the electrical system to fire an impulse through the cardiac ventricles and then recharge, also called repolarization. Longer QTc interval indicates prolonged repolarization, and when severe, may be a precursor to a specific form of deadly ventricular arrhythmia, Torsades de pointes (TdP). There were no episodes of TdP in bedaquiline treated patients, and no reported fatalities in patients with QTc prolongation, so the higher late mortality (in most cases many months following treatment completion) in patients receiving bedaquiline versus placebo in the small Phase II trial remains unexplained. Further evaluation of bedaquiline's risk-benefit profile in Phase III clinical trials is highly warranted. In addition, when bedaquiline is given with other QT-prolonging drugs, the effect on 
the QT interval is additive, complicating efforts to optimize multidrug treatment for MDR-TB.

\section{Verapamil co-administration potentiates bedaquiline action}

Our encouraging preclinical studies using the efflux inhibitor verapamil, prompted us to examine if addition of verapamil as adjunctive therapy with bedaquiline would help lower effective doses. We found that in vitro supplementation with verapamil profoundly decreased the MIC of bedaquiline among drug-susceptible and drug-resistant clinical isolates of M. $t b$ 8-16 fold [10]. Bedaquiline resistance conferred by mutations in the transcriptional repressor $R v 0678$, which upregulates the bacterial multisubstrate efflux pump MmpL5, demonstrates cross-resistance to clofazimine [11]; the effects of verapamil on clofazamine activity against these mutants is untested. We also investigated the effect of verapamil on bedaquiline's activity in the mouse model [12]. In the treatment of MDR-TB, bedaquiline is given at a dose of $400 \mathrm{mg}$ once daily for 2 weeks, followed by $200 \mathrm{mg}$ three times weekly for 22 weeks. Diacon et al. reported that the loading doses of bedaquiline were required to overcome the delayed antimicrobial activity observed in the patients [13]. To determine if verapamil may potentiate the activity of bedaquiline, in addition to the full human bioequivalent dose of bedaquiline (25 $\mathrm{mg} / \mathrm{kg}$ of body weight), we tested a suboptimal dose of bedaquiline $(12.5 \mathrm{mg} / \mathrm{kg})$ daily, with or without verapamil [12]. We found that over 6 weeks, co-administration of verapamil with 12.5 $\mathrm{mg} / \mathrm{kg}$ of bedaquiline gave the same bactericidal activity as $25 \mathrm{mg} / \mathrm{kg}$ of bedaquiline alone in mice. Adding verapamil to bedaquiline monotherapy also protected against the development of resistant mutants in vivo. In addition, we observed that in the presence of verapamil, bedaquiline showed bactericidal activity starting at week 1 , whereas in previous mouse and human studies, bactericidal activity was delayed [13]. Hence, use of verapamil may help reduce the bedaquiline loading doses required in the first 2 weeks of therapy. Our preclinical studies, therefore, indicate that verapamil potentiates the activity of bedaquiline against $M . t b$, reducing the bedaquiline dose required for cure. This potentiating effect may be due to efflux pump inhibition by verapamil, resulting in higher intracellular antimycobacterial drug levels and enhanced drug activity.

In addition to potentiating antibiotic-related killing by blocking efflux pumps, verapamil may also prevent tolerance. Whereas the frequency or generation of resistant mutants is in general less for bedaquiline than some first-line TB drugs, macrophage-induced tolerance to bedaquiline develops earlier than for isoniazid or rifampin in the setting of multidrug treatment (3). Inhibition of tolerance to bedaquiline is achieved with lower verapamil doses than those needed to inhibit tolerance to isoniazid.

\section{Verapamil may prevent QTc-associated cardiac arrhythmias}

In any multidrug therapy, drug-drug interactions and overlapping toxicities are critically important considerations, especially in the management of $\mathrm{TB}$, which requires prolonged multidrug treatment. Clinically-important drug-drug interactions are not predicted based on metabolic pathways of the two drugs. With regards to toxicities, while bedaquiline prolongs the QTc interval [9], verapamil has two effects on cardiac conduction: it extends the PR interval [14] and decreases the QTc interval. In fact, verapamil has been shown to be cardioprotective. It prevents TdP via shortening of endocardial monophasic ventricular action potential $[15,16]$. It abolishes early after-depolarization and reduces QT interval in both congenital and acquired long QT syndromes $[17,18]$. Verapamil completely suppresses $\mathrm{TdP}$ induced by dofetilide or by anticholinergic overdose $[19,20]$. Besides increasing the bactericidal activity of bedaquiline, it is therefore possible that the well-established protective effects of verapamil would limit or even abrogate the negative cardiac effects of bedaquiline.

Co-administration of verapamil with bedaquiline is a promising strategy for improving MDR-TB treatment efficacy and for reducing dose size requirements of bedaquiline; plus verapamil may be cardioprotective, reducing the risk of QT prolongation with bedaquiline and companion drugs. Further studies are, therefore, needed to investigate the synergistic effects of verapamil and bedaquiline in humans during TB infection.

\section{Financial \& competing interests disclosure}

The authors have received financial support from NIH grants A137856, AI36973 and AI097138, and from the Howard Hughes Medical Institute. The authors have no other relevant affiliations or financial involvement with any organization or entity with a financial interest in or financial conflict with the subject matter or materials discussed in the manuscript apart from those disclosed.

No writing assistance was utilized in the production of this manuscript. 


\section{References}

Papers of special note have been highlighted as: - of interest; $\bullet \bullet$ of considerable interest

1 Barry CE 3rd, Boshoff HI, Dartois V et al. The spectrum of latent tuberculosis: rethinking the biology and intervention strategies. Nat. Rev. Microbiol. 7(12), 845-855 (2009).

2 Adams KN, Takaki K, Connolly LE et al. Drug tolerance in replicating mycobacteria mediated by a macrophage-induced efflux mechanism. Cell 145(1), 39-53 (2011).

-• The study provides evidence for a novel mechanism of drug tolerance in mycobacteria that is induced by infection of macrophages and attributed to bacterial efflux pumps.

3 Adams KN, Szumowski JD, Ramakrishnan L. Verapamil, and its metabolite norverapamil, inhibit macrophage-induced, bacterial efflux pump-mediated tolerance to multiple anti-tubercular drugs. J. Infect. Dis. 210(3), 456-466 (2014).

- The study, following earlier work from the same laboratory, shows that verapamil reduces tolerance to isoniazid, rifampicin, bedaquiline and moxifloxacin in vitro.

4 Louw GE, Warren RM, Gey Van Pittius NC et al. Rifampicin reduces susceptibility to ofloxacin in rifampicin-resistant Mycobacterium tuberculosis through efflux. Am. J. Respir. Crit. Care Med. 184(2), 269-276 (2011).

- The study provides initial evidence that verapamil restores susceptibility to first-line drugs in mice infected with a MDR-TB strain.

5 Gupta S, Tyagi S, Almeida DV, Maiga MC, Ammerman NC, Bishai WR. Acceleration of tuberculosis treatment by adjunctive therapy with verapamil as an efflux inhibitor. Am. J. Respir. Crit. Care Med. 188(5), 600-607 (2013).

- The study shows that verapamil, when used as an adjunctive to standard chemotherapy, shortens treatment duration in a mouse model of tuberculosis.

6 Andries K, Verhasselt P, Guillemont J et al. A diarylquinoline drug active on the ATP synthase of Mycobacterium tuberculosis. Science 307(5707), 223-227 (2005).
- The study describes the discovery of a new antimycobacterial drug R207910 targeting ATP synthase of $M . t b$, and showing considerable antibacterial potency in vitro and in vivo.

7 Rustomjee R, Diacon AH, Allen J et al. Early bactericidal activity and pharmacokinetics of the diarylquinoline tmc207 in treatment of pulmonary tuberculosis. Antimicrob. Agents Chemother. 52(8), 2831-2835 (2008).

8 Diacon AH, Donald PR, Pym A et al. Randomized pilot trial of eight weeks of bedaquiline (tmc207) treatment for multidrug-resistant tuberculosis: long-term outcome, tolerability, and effect on emergence of drug resistance. Antimicrob. Agents Chemother. 56(6), 3271-3276 (2012).

- Exploratory double-blind randomized phase 2b study in newly diagnosed MDR-TB patients who received bedaquiline or placebo for 8 weeks in combination with a background second-line drug regimen showed reduced time for sputum culture conversion in patients receiving bedaquiline. An extended phase $2 b$ study by the same group [9] showed that the addition of bedaquiline to a background regimen for 24 weeks resulted in faster culture conversion in patients with MDR-TB, as compared with placebo.

9 Diacon AH, Pym A, Grobusch MP et al. Multidrug-resistant tuberculosis and culture conversion with bedaquiline. N. Engl. J. Med. 371(8), 723-732 (2014).

10 Gupta S, Cohen KA, Winglee K, Maiga M, Diarra B, Bishai WR. Efflux inhibition with verapamil potentiates bedaquiline in Mycobacterium tuberculosis. Antimicrob. Agents Chemother. 58(1), 574-576 (2014).

- The study shows that verapamil decreases the MIC of bedaquiline to $M . t b$ by 8-16-fold in vitro.

11 Andries K, Villellas C, Coeck N et al. Acquired resistance of Mycobacterium tuberculosis to bedaquiline. PLoS ONE 9(7), e102135 (2014).

12 Gupta S, Tyagi S, Bishai WR. Verapamil increases the bactericidal activity of bedaquiline against Mycobacterium tuberculosis in a mouse model. Antimicrob. Agents Chemother. 59(1), 673-676 (2015).
-. The study shows that co-administration of verapamil with subinhibitory doses of bedaquiline gives the same bactericidal effect in mouse model of TB as the full human bioequivalent dosing and that adding verapamil to bedaquiline monotherapy protects against the development of resistant mutants in vivo.

13 Diacon AH, Pym A, Grobusch M et al. The diarylquinoline tmc207 for multidrugresistant tuberculosis. N. Engl. J. Med. 360(23), 2397-2405 (2009).

14 Johnston A, Burgess CD, Hamer J. Systemic availability of oral verapamil and effect on PR interval in man. Br. J. Clin. Pharmacol. 12(3), 397-400 (1981).

15 Fauchier L, Babuty D, Poret P, Autret ML, Cosnay P, Fauchier JP. Effect of verapamil on Qt interval dynamicity. Am. J. Cardiol. 83(5), 807-808, A810-801 (1999).

16 Milberg P, Reinsch N, Osada N et al. Verapamil prevents torsade de pointes by reduction of transmural dispersion of repolarization and suppression of early afterdepolarizations in an intact heart model of lqt3. Basic Res. Cardiol. 100 (4), 365-371 (2005).

17 Shimizu W, Ohe T, Kurita T et al. Effects of verapamil and propranolol on early afterdepolarizations and ventricular arrhythmias induced by epinephrine in congenital long QT syndrome. J. Am. Coll. Cardiol. 26(5), 1299-1309 (1995).

18 Aiba T, Shimizu W, Inagaki M et al. Cellular and ionic mechanism for drug-induced long QT syndrome and effectiveness of verapamil. J. Am. Coll. Cardiol. 45(2), 300-307 (2005).

19 Oros A, Houtman MJ, Neco P et al. Robust anti-arrhythmic efficacy of verapamil and flunarizine against dofetilide-induced tdp arrhythmias is based upon a shared and a different mode of action. Br. J. Clin. Pharmacol. 161(1), 162-175 (2010).

20 Liao WB, Bullard MJ, Kuo CT, Hsiao CT, Chu PH, Chiang CW. Anticholinergic overdose induced torsade de pointes successfully treated with verapamil. Jpn. Heart J. 37(6), 925-931 (1996).

21 Annual Report of the National Institute for Research in Tuberculosis. http://www.nirt.res.in 